\title{
The weak hyperbolization conjecture for 3-dimensional CAT(0)-groups
}

\author{
Michael Kapovich and Bruce Kleiner
}

\begin{abstract}
We prove a weak hyperbolization conjecture for CAT(0) 3-dimensional Poincaré duality groups.
\end{abstract}

Mathematics Subject Classification (2000). Primary 20F65, 20F67; Secondary 57P10.

Keywords. Poincaré duality groups; CAT(0)-groups.

\section{Introduction}

For a variety of classes of groups, it is a well-known open problem whether the failure of Gromov hyperbolicity can be detected by the presence of special subgroups, e.g. rank 2 abelian groups or Baumslag-Solitar groups. This is of interest, for instance, for CAT(0)-groups (even for the fundamental groups of finite 2-dimensional locally CAT(0) square complexes), for 1-relator groups, and 3-dimensional Poincaré duality groups. We say that a class of groups satisfies the weak hyperbolization conjecture if every group in the class is either Gromov hyperbolic, or contains a copy of $\mathbb{Z}^{2}$. We recall that the weak hyperbolization conjecture for 3-manifold groups was a part of the program for proving the Geometrization Conjecture for closed irreducible aspherical 3-manifolds, the other ingredient in the program being the Cannon conjecture. Although the work of Perelman has now resolved the full Geometrization Conjecture, the weak hyperbolization conjecture for $\mathrm{PD}(3)$-groups is a potential step in an approach to the following open question of C.T.C. Wall:

Question 1 (Wall). Is every finitely presented $\operatorname{PD(3)-group~over~} \mathbb{Z}$ isomorphic to the fundamental group of a closed aspherical 3-manifold?

Our main result is that the weak hyperbolization conjecture holds for CAT(0) 3-dimensional Poincaré duality groups over hereditary rings: 
Theorem 2. Let $G$ be a 3-dimensional Poincaré duality group over a commutative hereditary ring $\mathcal{R}$ with a unit. Suppose in addition that $G$ is a $\mathrm{CAT}(0)$-group, i.e., a group which admits a cocompact isometric properly discontinuous action $G \curvearrowright X$ on a locally compact CAT(0) space $X$.

Then $G$ satisfies the weak hyperbolization conjecture.

We refer the reader to [9] for the definition of a hereditary ring; here we note only that every PID is hereditary.

We note that special cases of this theorem were proven earlier by various people: S. Buyalo [8] and V. Schroeder [18] independently have proven that this theorem holds provided that $X$ is the universal cover $\tilde{M}$ of a closed 3-manifold $M$, the CAT(0) structure on $\tilde{M}$ is Riemannian and $G=\pi_{1}(M)$ acts on $X$ by deck-transformations. L. Mosher [16] proved that Theorem 2 holds provided that $X=\tilde{M}, G=\pi_{1}(M)$, and the CAT(0) metric on $X$ is obtained by lifting a piecewise-Euclidean (locally) CAT(0)-cubulation from $M$. M. Bridson and L. Mosher also have an unpublished proof of Theorem 2 under the assumption that $X=\tilde{M}$ has an arbitrary $G$-invariant CAT(0) structure. Unlike all these proofs, our proof takes place on the ideal boundary of $X$; this allows us to treat 3-dimensional Poincaré duality groups and relax the assumptions on the CAT( 0$)$ space.

Outline of the proof of Theorem 2. Assume that $G$ is not Gromov hyperbolic, i.e., that $X$ contains a 2-flat. By the work of Bestvina [2], the ideal boundary of $X$ is homeomorphic to $S^{2}$. Our proof exploits the geometry of flats and parallel sets in $X$, and the pattern of their boundaries in the 2-sphere $\partial_{\infty} X$. The proof breaks into three cases.

Case 1. $X$ contains a 3-flat, Section 5.1. This implies that $X$ is at finite Hausdorff distance from the 3 -flat, and we conclude that $G$ is virtually $\mathbb{Z}^{3}$.

Case 2. $X$ contains no 3-flat but some parallel set $P \subset X$ has full ideal boundary, i.e. $\partial_{\infty} P=\partial_{\infty} X$, Section 5.3. We argue that $P$ splits isometrically as $\mathbb{R} \times Y$, where $\partial_{\infty} Y$ is a circle, and $G$ acts as a convergence group on $\partial_{\infty} Y$. We then deduce that a finite index subgroup of $G$ is isomorphic to the fundamental group of a 3-dimensional Seifert manifold.

Case 3. X contains no 3-flat and no parallel set with full boundary, Section 5.4. This is the main case. We show that every parallel set $P$ in $X$ is isometric to a product $\mathbb{R} \times Y$, where $Y$ is Gromov hyperbolic. The ideal boundary of $P$ is a suspension of the boundary $\partial_{\infty} Y$; when $P$ contains a 2 -flat, we identify certain topological circles in $\partial_{\infty} P$ which we call peripheral, and show that peripheral circles cannot cross one another in the 2-sphere $\partial_{\infty} X$.

Next, we choose a flat $F \subset X$ whose boundary $\partial_{\infty} F \subset \partial_{\infty} X$ is a peripheral circle, and consider its orbit $\{g(F)\} g \in G$. Because the circles $\left\{g\left(\partial_{\infty} F\right)\right\} g \in G$ do not cross, we may use them to define a pretree $\mathcal{T}$ on which $G$ has a natural action. Using a Plante-type construction, we associate to $\mathcal{T}$ an $\mathbb{R}$-tree $T$, which then inherits a 
nontrivial small stable $G$-action. By applying Rips' theory [3], we conclude that $G$ admits a small nontrivial action on a simplicial tree. Using the fact that $G$ is a $\operatorname{PD(3)-~}$ group, we deduce that the edge groups must be virtually $\mathbb{Z}^{2}$.

Acknowledgements. The first author was supported in part by NSF Grants DMS-0203045 and DMS-04-05180, the second author was supported in part by NSF Grants DMS-02-24104 and DMS-05-05610. The authors are grateful to the referee for useful suggestions.

\section{Geometric preliminaries}

In this section we briefly review several notions of metric geometry. We refer the reader to [1], [6] for the detailed discussion.

A geodesic metric space is a metric space $(X, d)$ such that any two points $x, y \in X$ in $X$ are connected by geodesic, i.e., if $D:=d(x, y)$ then there exists an isometric embedding

$$
\gamma:[0, D] \rightarrow X
$$

so that $\gamma(0)=x, \gamma(D)=y$.

Let $X$ be a metric space and $C \subset X$ be a subset. The $r$-neighborhood of $C$ in $X$ is defined as

$$
N_{r}(C):=\{x \in X: d(x, C)<r\},
$$

where $d(x, C):=\inf \{d(x, c): c \in C\}$.

The Hausdorff distance between closed subsets of a metric space $X$ is defined as

$$
d_{H}\left(C_{1}, C_{2}\right):=\inf \left\{r: C_{1} \subset N_{r}\left(C_{2}\right), C_{2} \subset N_{r}\left(C_{1}\right)\right\} .
$$

Note that this distance is allowed to take infinite values. If $X$ has finite diameter, the Hausdorff distance defines the Hausdorff topology on the set $\mathcal{C}(X)$ of closed subsets of $X$. More generally, even for unbounded metric spaces $X$ one defines the Gromov-Hausdorff topology on $\mathcal{C}(X)$ as follows. We say that a sequence $C_{n} \in \mathcal{C}(X)$ converges (in the Gromov-Hausdorff topology) to a closed set $C \in \mathcal{C}(X)$ if for each closed metric ball $B \subset X$ the intersections

$$
C_{n} \cap B \in \mathcal{C}(B)
$$

converge to $C \cap B$ in the Hausdorff topology on $C(B)$. Equivalently, $C_{n}$ 's converge to $C$ if the corresponding distance functions $d\left(\cdot, C_{n}\right)$ converge to the distance function $d(\cdot, C)$ uniformly on bounded subsets in $X$.

Given a number $\kappa \in \mathbb{R}$ let $M_{\kappa}$ denote the (unique up to isometry) complete simply-connected surface of the constant curvature $\kappa$. A geodesic metric space $X$ is said to be a $\mathrm{CAT}(\kappa)$ space if $X$ is complete as a metric space and geodesic triangles 
in $X$ are "thinner" than triangles in $M_{\kappa}$. More precisely, consider a geodesic triangle $T=[x, y, z] \subset X$ (with the vertices $x, y, z$ ), in case when $\kappa>0$ (and $M_{\kappa}$ is a sphere) we assume that the perimeter of this triangle is less than the circumference of the great circle in $M_{\kappa}$. Consider a triangle $T^{\prime}=\left[x^{\prime}, y^{\prime}, z^{\prime}\right] \subset M_{\kappa}$ whose side-lengths are equal to the corresponding side-lengths of the triangle $T$. Let $p$ be a point in the geodesic side $\overline{x y}$ of $T$ and let $p^{\prime} \in \overline{x^{\prime} y^{\prime}}$ be such that

$$
d\left(x^{\prime}, p^{\prime}\right)=d(x, p)
$$

Then we require

$$
d(z, p) \leq d\left(z^{\prime}, p^{\prime}\right) .
$$

In this paper we will also need a generalization of the concept of a CAT(1) space to metric spaces $X$ which are not geodesic. We assume that $X$ is a disjoint union of geodesic metric spaces $X_{\alpha}, \alpha \in J$, where each $X_{\alpha}$ is a geodesic CAT(1) metric space and if $\alpha \neq \beta$ the distance between any $x \in X_{\alpha}, y \in X_{\beta}$ equals $\pi$. Then $X$ will be also referred to as a CAT(1) space. An example of such a space is a space with discrete metric where distance between any pair of distinct points equals $\pi$.

If $X$ is a CAT(1) space, we call points $x, y \in X$ antipodal if $d(x, y)=\pi$.

Suppose that $X$ is a $\operatorname{CAT}(0)$ space. Then the distance function on $X$ is convex, i.e., its restriction to each geodesic in $X$ is convex.

A space $X$ is called $\operatorname{CAT}(-\infty)$ if it is $\operatorname{CAT}(\kappa)$ for each $\kappa \in \mathbb{R}$. A metric tree is a $\mathrm{CAT}(-\infty)$; in other words, it is a complete geodesic metric space where each geodesic triangle is isometric to a tripod.

A group $G$ is called a CAT(0)-group if it admits an isometric properly discontinuous cocompact action on a locally compact $\mathrm{CAT}(0)$ space.

Suppose that $X$ is a CAT(0) space and $F \subset X$ is a $k$-flat, i.e., an isometrically embedded copy of a Euclidean space $\mathbb{R}^{k}$. Then the parallel set $P_{F}$ of $F$ in $X$ is the union of all $k$-flats $F^{\prime} \subset X$ which are within finite distance from $F$. The parallel set $P_{F}$ is closed, convex and is isometric to a product

$$
F \times Y
$$

where $Y$ is a $\mathrm{CAT}(0)$ space, see for instance [6, Theorem II.2.14].

Remark 3. Theorem II.2.14 in [6] is stated in the case $k=1$. The general case follows, for instance, by induction on the dimension of the flat.

We will say that a parallel set is trivial if $k=1$ and $Y$ is bounded.

Given a CAT(0) space one defines the ideal boundary of $X$ as the collection of equivalence classes of geodesic rays in $X$, where rays are equivalent if they are within finite Hausdorff distance from each other. This boundary has two (typically distinct) topologies: 
1. the visual topology, in which case the ideal boundary is denoted $\partial_{\infty} X$ and is called the geometric boundary of $X$;

2. the Tits topology, which is defined via the Tits angular metric, in which case the ideal boundary is denoted $\partial_{\text {Tits }} X$.

The second boundary is called Tits boundary of $X$; this boundary is always a CAT(1) space.

For instance, in the case when $X=\mathbb{H}^{2}, \partial_{\infty} X$ is homeomorphic to $S^{1}$, while $\partial_{\text {Tits }} X$ has discrete metric: the distance between distinct points equals $\pi$. A CAT(0) space is called a visibility space if any pair of distinct points in $\partial_{\text {Tits }} X$ are antipodal.

A subset $C \subset Z:=\partial_{\text {Tits }} X$ is called convex if for any two non-antipodal points $x, y \in Z$, the geodesic segment $\overline{x y}$ connecting $x$ to $y$, is entirely contained in $C$. Intersection of two convex subsets of $Z$ is also convex. If $Y \subset X$ is a convex subset then $\partial_{\text {Tits }} Y \subset Z$ is convex as well.

Let $\delta \in[0, \infty)$ and consider a geodesic metric space $X$. A triangle $T \subset X$ is called $\delta$-thin if there exists a point $p \in X$ which is within distance $\leq \delta$ from all three sides of $T$. A complete geodesic metric space $X$ is called $\delta$-hyperbolic if each geodesic triangle $T$ in $X$ is $\delta$-thin. A space $X$ is called Gromov-hyperbolic if it is $\delta$-hyperbolic for some $\delta$. A finitely generated group $G$ is called Gromov-hyperbolic if its Cayley graph is Gromov-hyperbolic. One again defines the ideal boundary $\partial_{\infty} X$ by looking at the equivalence classes of geodesic rays in $X$.

Suppose that $G$ is a group acting isometrically, properly discontinuously and cocompactly on a CAT( 0$)$ space $X$. Then the group $G$ is Gromov-hyperbolic iff $X$ is a visibility space.

Let $X$ be a Gromov-hyperbolic geodesic metric space which admits a cocompact isometric group action. We assume that the ideal boundary of $X$ consists of more than 2 points; it then follows that $\partial_{\infty} X$ has the cardinality of the continuum. The displacement function of an isometry $g: X \rightarrow X$ is

$$
\operatorname{dis}(g): x \rightarrow d(x, g(x)), \quad x \in X .
$$

Lemma 4. Under the above assumptions there exists a constant $D=D(X)$ such that for each $g \in \operatorname{Isom}(X)$ which fixes $\partial_{\infty} X$ pointwise, the displacement of $g$ is bounded from above by $D$.

Proof. Let $G \curvearrowright X$ be a cocompact isometric group action; pick a metric ball $B=$ $B(o, R) \subset X$ so that the $G$-orbit of $B$ equals $X$. It then suffices to prove that there exists $D<\infty$ such that for each isometry $g$ of $X$ fixing $\partial_{\infty} X$ pointwise,

$$
d(o, g(o)) \leq D .
$$

Since the ideal boundary of $X$ contains at least 4 points, there exists a pair of geodesics $\gamma_{1}, \gamma_{2} \subset X$ which have disjoint ideal boundaries. Without loss of generality we may assume that both $\gamma_{1}, \gamma_{2}$ pass through the ball $B$. 
Since $X$ is $\delta$-hyperbolic, there exists a number $r=r(\delta)<\infty$ such that if geodesics $\alpha, \beta \subset X$ are within finite Hausdorff distance, then

$$
d_{H}(\alpha, \beta) \leq r,
$$

see for instance [6]. For every isometry $g$ as above, the geodesics

$$
\gamma_{i}, g\left(\gamma_{i}\right)
$$

are within finite Hausdorff distance from each other; therefore

$$
d_{H}\left(\gamma_{i}, g\left(\gamma_{i}\right)\right) \leq r, \quad i=1,2 .
$$

Then

$$
d\left(g(o), g\left(\gamma_{i}\right)\right) \leq R \Longrightarrow d\left(g(o), \gamma_{i}\right) \leq R+r, \quad i=1,2 .
$$

However, since the geodesics $\gamma_{1}, \gamma_{2}$ have disjoint ideal boundaries, the diameter of

$$
S:=N_{R+r}\left(\gamma_{1}\right) \cap N_{R+r}\left(\gamma_{2}\right)
$$

is finite. Therefore, if we take $D:=\operatorname{diam}(S) / 2$, the distance between $o$ and $g(o)$ is at most $D$.

Remark 5. An analogue of Lemma 4 holds for quasi-isometries of $X$ with uniformly bounded quasi-isometry constants.

\section{Pretrees}

In what follows we will need definitions and basic facts about pretrees; the definitions which we give follow [5].

A pretree is a set $T$ together with a ternary relation (the betweenness relation)

$$
\text { " } y \text { is between } x \text { and } z \text { ", }
$$

to be denoted $\beta(x y z)$, satisfying the following axioms:

Axiom 1. $\beta(x y z)$ implies that $x \neq y \neq z$.

Axiom 2. $\beta(x y z) \Longleftrightarrow \beta(z y x)$.

Axiom 3. $\beta(x y z)$ and $\beta(y x z)$ cannot hold simultaneously.

Axiom 4. If $w \neq y$ then $\beta(x y z)$ implies that either $\beta(x y w)$ or $\beta(w y z)$.

Given a pretree $T$ one can define closed, open and half-open intervals in $T$ by

$$
(x, z):=\{y \in T: \beta(x y z)\}, \quad[x, z]:=(x, z) \cup\{x, z\}, \text { etc. }
$$


Given an increasing union of intervals

$$
\left[x_{1}, y_{1}\right] \subset\left[x_{2}, y_{2}\right] \subset \cdots \subset\left[x_{i}, y_{i}\right] \subset \cdots
$$

we will also refer to the union of these intervals as a (possibly infinite) interval in $T$.

We note that $\beta$ defines an order on each interval in $T$.

Define a "triangle" in $T$ with vertices $a, b, c$ to be the union of the segments (called "sides" of the triangle) $[a, b],[b, c],[c, a]$.

Lemma 6. Each triangle $\Delta$ in $T$ is 0 -thin, i.e., each side of $\Delta$ is contained in the union of the two other sides.

Proof. Follows immediately from Axiom 4.

Suppose that $T$ is a pretree which is given a measure $\mu$ (without atoms) defined on closed intervals in $T$ and the $\sigma$-algebra which these intervals generate. Define a function $d(x, y)$ on $T$ by $d(x, y):=\mu([x, y])$.

Lemma 7. $d$ is a pseudo-metric on $T$.

Proof. It is clear that $d$ is symmetric and $d(x, x)=0$ (since $\mu$ has no atoms). The triangle inequality follows because for each triangle with the vertices $a, b, c$ we have (see Lemma 6)

$$
[a, b] \subset[a, c] \cup[b, c] .
$$

We note that if for each interval $[a, b] \subset T$, with $a \neq b, \mu(a, b)>0$ then $d$ is a metric. Moreover, it follows that $(a, b) \neq \varnothing$ for each $a \neq b$. If the restriction of the metric $d$ to each interval $[x, y]$ is complete then $[x, y]$ is order isomorphic to an interval in $\mathbb{R}$ and moreover, $([x, y], d)$ is isometric to an interval in $\mathbb{R}$. We thus get:

Lemma 8. Suppose that for each interval $[x, y] \subset T$, with $x \neq y, \mu[x, y]>0$, and that the restriction of the metric $d$ to each interval in $T$ is complete. Then $(T, d)$ is a metric tree.

Proof. It is clear from the above discussion that $T$ is a geodesic metric space. Since each triangle in $T$ is 0 -thin, it follows that each triangle in $T$ is isometric to a tripod. Finally, let us check completeness of $T$ : Suppose that $x_{i}, i \geq 0$, is a Cauchy sequence in $T$. Then there exists an increasing sequence of intervals $I_{i} \subset T$ such that

$$
\lim _{i} \mu\left(\left[x_{0}, x_{i}\right] \cap I_{i}\right)=\lim _{i} d\left(x_{0}, x_{i}\right) .
$$

Then completeness of $d$ restricted to the union $I$ of $I_{i}$ 's implies that $\left(x_{i}\right)$ converges to a point in the interval $I$. 


\section{Ideal boundaries of CAT(0) Poincaré duality groups}

Let $G \curvearrowright X$ be a discrete cocompact action of a PD(3)-group $G$ on a CAT(0) space $X$. In this section we show that the ideal boundary of the CAT(0) space $X$ is homeomorphic to $S^{2}$.

We refer the reader to [4], [7] for the background on the cohomology of groups. Recall [4] that an $n$-dimensional Poincaré duality group over a ring $\mathcal{R}$ (for short, $\operatorname{PD}(n)$-group over $\mathcal{R})$, is an FP-group over $\mathcal{R}$ such that $H^{i}(G, \mathcal{R} G)$ is isomorphic to $\mathcal{R}$ as an $\mathcal{R}$-module when $i=n$ and is trivial otherwise.

Let $Z:=\partial_{\infty} X$ be the ideal boundary of a locally compact CAT(0) space. M. Bestvina in [2] proved that the compactification

$$
\bar{X}:=X \cup Z
$$

satisfies the axioms of the $\mathbb{Z}$-set compactification. Instead of listing all the axioms of the $Z$-set compactification we mention only several properties:

1. If $G \curvearrowright X$ is an isometric group action then this action extends to a topological action of $G$ on $\bar{X}$.

2. There exists a natural isomorphism

$$
H_{c}^{*}(X) \rightarrow \tilde{H}_{c}^{*-1}(Z),
$$

which is compatible with inclusions of closed convex subsets $X^{\prime} \subset X$.

3. We state the third property as a lemma:

Lemma 9. If $G$ is a $\mathrm{PD}(3)$-group acting isometrically, properly discontinuously and cocompactly on a $\mathrm{CAT}(0)$ space $X$, then the ideal boundary $Z$ of $X$ is homeomorphic to $S^{2}$.

Proof. Bestvina proves, [2, Theorem 2.8], that if $G$ is a $\mathrm{PD}$ (3)-group over $\mathcal{R}$, then $Z$ is homeomorphic to $S^{2}$. We note that Bestvina proves the latter theorem under more restrictive assumptions than we are working with (although, his class of groups $G$ includes 3-manifold groups):

1. Bestvina assumes that the commutative ring $\mathcal{R}$ is a PID. However this assumption is used only to apply the Universal Coefficients Theorem, which works for hereditary rings as well, see [9].

2. Bestvina's definition of an $n$-dimensional Poincaré duality group is more restrictive than the usual one: Instead of the FP-property he assumes that a group $G$ acts freely, properly discontinuously, cocompactly on a contractible cell complex $Y$. Note however that Bestvina in his proof uses only the fact that $G \curvearrowright Y^{(i)}$ is cocompact on each $i$-skeleton of $Y$. Then existence of such an action for the CAT( 0$)$-groups follows from a general construction described in [14]. Namely, if a group $G$ admits a properly discontinuous cocompact action on a contractible space $X$ (e.g. the CAT(0) space in 
our case) then it also admits a free, properly discontinuous action on a contractible cell complex $Y$ (possibly of infinite dimension) such that $Y^{(i)} / G$ is compact for each $i$.

3. Bestvina assumes that the image of the orientation character $\chi$ of the Poincaré duality group $G$ is finite (he then passes to a finite index subgroup in $G$ which is the kernel of $\chi$ ). However this assumption can be omitted from his theorem using twisting of the action $G \curvearrowright C_{*}(Y)$ by the character $\chi$ as it is done in [14, Section 5.1].

With the above modifications, Bestvina's arguments apply in our case and it follows that $\partial_{\infty} X$ is homeomorphic to the 2 -sphere.

\section{Proof of the main theorem}

5.1. Case 1: $X$ contains a 3-flat. The main goal of this section is to show that, in case $X$ contains a 3 -flat, the group $G$ contains a finite index subgroup isomorphic to $\mathbb{Z}^{3}$.

Lemma 10. Suppose that $S$ is a convex subset in $X$ such that $\partial_{\infty} S=\partial_{\infty} X$. Then $S$ is within finite Hausdorff distance from $X$.

Proof. Pick a base-point $o \in X$. If $S$ is not within finite Hausdorff distance from $X$ then there exists a sequence of isometries $g_{i} \in G$ such that $d\left(o, g_{i} S\right)$ diverges to infinity. Consider the functions $f_{i}:=d\left(x, g_{i} S\right)-d\left(o, g_{i} S\right)$. Then, according to Lemma 2.3 in [15], the sequence of functions $f_{i}$ subconverges to a Busemann function $b$ on $X$. Clearly, the sublevel sets $\left\{f_{i} \leq 0\right\}$ subconverge into the horoball $U:=\{b \leq 0\}$ in $X$. Since $\partial_{\infty}\left\{f_{i} \leq 0\right\}=\partial_{\infty} g_{i} S=\partial_{\infty} X$, it follows that $\partial_{\infty} X=\partial_{\infty} U$.

Let $F$ be a 2-flat in $X$. Then $\partial_{\infty} F \subset \partial_{\infty} U$ and the convexity of horoballs in $X$ imply that for each $x \in F$,

$$
t=f(x) \Longrightarrow F \subset\{z: b(z) \leq t\}
$$

It follows that the restriction $b \mid F$ is constant and thus $F$ is contained in the horosphere $\{x: b(x)=t\}$ for some $t \in \mathbb{R}$. Then Lemma 2.2 in [15] implies that $X$ contains a half-space $H:=\mathbb{R}_{+} \times F$. Then, by taking an appropriate limit of the half-spaces $h_{j}(H), h_{j} \in G$, we see that $X$ contains the 3-flat $F^{\prime}:=F \times \mathbb{R}$. By Lemma 9, $\partial_{\infty} F^{\prime}=\partial_{\infty} X$. Suppose that $F^{\prime}$ is not within finite Hausdorff distance from $X$. Then, by repeating the same argument as above with $S$ replaced with $F^{\prime}$ and then $F$ replaced with $F^{\prime}$, we see that $X$ contains a 4-flat, which contradicts Lemma 9.

Therefore $X$ is within finite Hausdorff distance from the 3-flat $F^{\prime}$; in particular, there are no horoballs in $X$ which have the same ideal boundary as $X$. Contradiction. 
Corollary 11. If $X$ contains a 3 -flat then the group $G$ is virtually abelian; in particular, it contains $\mathbb{Z} \times \mathbb{Z}$.

Proof. If $F$ is a 3-flat in $X$ then, by Lemma $9, \partial_{\infty} F=\partial_{\infty} X$ and, by Lemma $10, F$ is within finite Hausdorff distance from $X$. It follows that the group $G$ is isomorphic to a lattice in $\operatorname{Isom}\left(\mathbb{R}^{3}\right)$ and hence it is virtually abelian and contains $\mathbb{Z}^{3}$ as a subgroup of finite index.

Assumption. From now on we will assume that $X$ contains no 3-flats.

5.2. Metric balls and parallel sets in $X$. In this section we establish certain geometric properties of $X$ which follow from the above assumption.

Lemma 12. There exists $r_{0} \in \mathbb{R}$ such that the following holds. For each ball $B(x, r) \subset X$, isometric to a disk of the radius $r$ in $\mathbb{R}^{3}$, we have $r \leq r_{0}$.

Proof. If the assertion is false then there exists a sequence of balls $B\left(x_{i}, r_{i}\right)$ with $\lim _{i} r_{i}=\infty$. Let $g_{i} \in G$ be such that $g_{i}\left(x_{i}\right)$ is a bounded sequence in $X$. Then the balls $g_{i}\left(B\left(x_{i}, r_{i}\right)\right)$ subconverge to a 3 -flat in $X$. Contradiction.

Corollary 13. The set of 2-flats $F^{\prime} \subset X$ which are parallel to a flat $F$ is compact in the Gromov-Hausdorff topology.

Proof. If not then $X$ contains convex subsets isometric to $[0, r] \times \mathbb{R}^{2}$ for arbitrarily large $r$. This contradicts the previous lemma.

Lemma 14. Suppose that $Y \times \mathbb{R}$ is a parallel set in $X$. Then $Y$ is Gromov-hyperbolic.

Proof. We repeat the arguments in [6, Theorem 9.33]. If $Y$ is not Gromov-hyperbolic then there exists a pair of points $\xi, \eta \in \partial_{\infty} Y$ so that the Tits angle between $\xi, \eta$ is positive but less than $\pi$. Pick a point $o \in Y$ and consider a sequence of points $y_{i} \in \overline{o \xi}$ which converge to $\xi$ and the geodesic rays $\overline{y_{i} \eta}$. We identify the rays $\overline{y_{i} \xi}, \overline{y_{i} \eta}$ with geodesic rays in $Y \times \mathbb{R} \subset X$ (that share common point $y_{i}$ ). Then, by applying an appropriate sequence of elements $g_{i} \in G$ (for which $\left\{g_{i}\left(y_{i}\right)\right\}$ is bounded in $X$ ) to $Y \times \mathbb{R}$ and to the rays $\overline{y_{i} \xi}, \overline{y_{i} \eta}$ and passing to the limit of a subsequence, we get:

1 . The sets $g_{i}(Y \times \mathbb{R})$ subconverge to a parallel set $Y^{\prime} \times \mathbb{R}$.

2. $Y^{\prime}$ contains two geodesic rays $\overline{y \xi^{\prime}}, \overline{y \eta^{\prime}}$ (limits of the sequences of rays $g_{i}\left(\overline{y_{i} \xi}\right)$, $\left.g_{i}\left(\overline{y_{i} \eta}\right)\right)$ which bound a flat sector in $Y^{\prime}$.

This contradicts Lemma 12.

5.3. Case 2: $X$ contains a parallel set with the full boundary. In this section we prove the main theorem under the assumption that $X$ contains a parallel set $P$ whose ideal boundary is the entire $\partial_{\infty} X$. 
Proposition 15. Suppose that there is a convex product subset $P=\mathbb{R} \times Y$ such that $\partial_{\infty} S=\partial_{\infty} X$. Then $G$ is commensurable to the fundamental group of a 3-dimensional Seifert manifold. In particular, $G$ contains $\mathbb{Z}^{2}$.

Proof. We will assume that $P$ is a maximal convex product subset in $X$. Since $Y$ is Gromov-hyperbolic, it follows that the Tits boundary of $S$ is the suspension of a discrete metric space which is the ideal boundary of $Y$. Therefore, since $\partial_{\infty} P=$ $\partial_{\infty} X$, the group $G$ preserves the ideal boundary of the geodesic $l=\mathbb{R} \times\{y\}$. Hence for each $g \in G$ the geodesic $g(l)$ is parallel to $l$, which (by the maximality assumption) implies that $g(P)=P$.

We have an induced isometric action $\rho: G \curvearrowright Y$. Since the suspension of $\partial_{\infty} Y$ is homeomorphic to the 2-sphere $\partial_{\infty} X$, the ideal boundary of $Y$ is homeomorphic to $S^{1}$. Thus the cocompact isometric action $\rho: G \curvearrowright Y$ extends to a uniform (topological) convergence action $G \curvearrowright \partial_{\infty} Y=S^{1}$. Therefore, according to [10], [12], [13], [19], the action $G \curvearrowright S^{1}$ is topologically conjugate to a Moebius action $\rho^{\prime}$.

Let $K$ denote the kernel of $\rho^{\prime}$.

Lemma 16. $K$ contains an infinite cyclic subgroup of finite index.

Proof. Let $D=D(Y)$ denote the constant given by Lemma 4. Pick a point $y \in Y$. Then for each $g \in K$,

$$
d(y, g(y)) \leq D
$$

Therefore the $K$-orbit of $y$ is contained in the metric ball $B(y, D)$. Thus for every $x \in X$, the $K$-orbit of $x$ is contained in a $D$-neighborhood of the geodesic $l=\{y\} \times \mathbb{R}$ (passing through $x$ ). Therefore $K$ is quasi-isometric to $\mathbb{Z}$ and hence is virtually $\mathbb{Z}$.

Lemma 17. The action $G \curvearrowright S^{1}$ is topologically conjugate to an action of a uniform lattice in $\operatorname{Isom}\left(\mathbb{H}^{2}\right)$.

Proof. The action $\rho^{\prime}(G) \curvearrowright \mathbb{H}^{2}$ is cocompact, therefore we have the following possibilities:

(a) $\rho^{\prime}(G)$ is a cocompact discrete subgroup in $\operatorname{Isom}\left(\mathbb{H}^{2}\right)$.

(b) $\rho^{\prime}(G)$ is a solvable subgroup in $\operatorname{Isom}\left(\mathbb{H}^{2}\right)$, which fixes a point in $S^{1}$. Then $\rho^{\prime}(G)$ is not virtually abelian which contradicts the fact that $G$ is a CAT(0)-group.

(c) $\rho^{\prime}(G)$ is dense in $\operatorname{PSL}(2, \mathbb{R})$. Then, the group $\rho^{\prime}(G)$ contains a nontrivial elliptic element $\hat{g}$ and it also contains a sequence of elements $\hat{h}_{i}$ which converge to $1 \in \operatorname{PSL}(2, \mathbb{R})$. Let $g, h_{i} \in G$ be elements which map (via $\rho^{\prime}$ ) to $\hat{g}$ and $\hat{h}_{i}$ respectively. Clearly, $\rho(g) \in \operatorname{Isom}(Y)$ is elliptic as well, let $y \in Y$ be its fixed point. By taking conjugates $g_{i}:=h_{i} g h_{i}^{-1}$, we get an infinite collection of distinct elements $\left\{g_{i}: i \in \mathbb{N}\right\}$ of $G$ such that for each $n \in \mathbb{Z}, g_{i}(y \times \mathbb{R})$ is contained in $N_{R}(y \times \mathbb{R})$ where $R \in \mathbb{R}_{+}$is independent of $i$. We note that since all $g_{i}$ are pairwise conjugate, 
there exists $C<\infty$ such that $d\left(x, g_{i}(x)\right)<C$ for each $x \in y \times \mathbb{R}$ and $i \in \mathbb{N}$. This contradicts discreteness of the action of $G$ on $X$.

The above two lemmas imply that the kernel of $\rho$ is commensurable to $\mathbb{Z}$ and the quotient $\rho(G)$ is commensurable to the fundamental group of a 2-dimensional hyperbolic surface. Thus, after passing to a finite index subgroup in $G$ we obtain a short exact sequence

$$
1 \rightarrow K \rightarrow G \rightarrow Q \rightarrow 1
$$

where $Q$ is the fundamental group of a closed oriented surface.

Lemma 19. Suppose that for a group $H$ we have a short exact sequence

$$
1 \rightarrow \mathbb{Z} / n \mathbb{Z} \rightarrow H \rightarrow Q \rightarrow 1 .
$$

Then $H$ contains a finite index surface subgroup.

Proof. Let $t$ denote the generator of $\mathbb{Z} / n \mathbb{Z}$. Let $a_{i}, b_{i}, i=1, \ldots, n$, denote the lifts to $H$ of the standard generators of $Q$. It suffices to consider the case when

$$
\left[a_{1}, b_{1}\right] \ldots\left[a_{n}, b_{n}\right]=t
$$

and $t$ belongs to the center of $H$. Consider the finite Heisenberg group

$$
H_{n}:=\left\langle a, b, t:[a, b]=t, a^{n}=b^{n}=t^{n}=1,[a, t]=1,[b, t]=1\right\rangle .
$$

Define the homomorphism $\phi: H \rightarrow H_{n}$ by

$$
\phi\left(a_{1}\right)=a, \quad \phi\left(b_{1}\right)=b, \quad \phi\left(a_{i}\right)=\phi\left(b_{i}\right)=1 \quad \text { for all } i \geq 2 .
$$

Then the kernel $H^{\prime}$ of $\phi$ is a torsion-free subgroup of finite index in $H$. It follows that the map $H \rightarrow Q$ sends $H^{\prime}$ injectively to a finite index subgroup in $Q$. Therefore $H^{\prime}$ is a surface group.

We now return to the exact sequence (18). As in the above lemma we let $a_{i}, b_{i}$, $i=1, \ldots, n$, denote the lifts to $G$ of the standard generators of $Q$. Let $H \subset G$ denote the subgroup generated by these elements. If

$$
t:=\left[a_{1}, b_{1}\right] \ldots\left[a_{n}, b_{n}\right]
$$

is an infinite order element of $K$ then $H$ is isomorphic to the fundamental group of a Seifert manifold (whose base is a surface with the fundamental group $Q$ ). It is clear that $H$ has finite index in $G$.

It $t$ has finite order then, according to Lemma 19, after passing to a finite index subgroup in $Q$ ) we can assume that $t=1$. Pick an infinite order element $k \in K$ which belongs to the center of $G$. Then the subgroups $H$ and $\langle k\rangle$ generate the product

$$
\mathbb{Z} \times Q \subset G
$$


Again, clearly, this subgroup has finite index in $G$. Thus, in the both cases, $G$ is commensurable to the fundamental group of a 3-dimensional Seifert manifold.

Thus, the conclusion of Theorem 2 holds provided that $X$ contains a parallel set with the full boundary.

Assumption. From now on we will assume that the ideal boundary of each parallel set of $X$ is a proper subset of $\partial_{\infty} X$.

\subsection{Case 3: The ideal boundary of every parallel set in $X$ is a proper subset of}

$\partial_{\infty} \boldsymbol{X}$. In this section we show that the peripheral circles of the ideal boundaries of nontrivial parallel sets in $X$ can be used to construct a small stable nontrivial isometric action of $G$ on an $\mathbb{R}$-tree. Then, by Rips theory, $G$ admits a nontrivial splitting as an amalgam with virtually abelian edge groups. This, in turn, implies that the edge groups are virtually $\mathbb{Z}^{2}$.

According to Eberlein's theorem (see [11] in the smooth case and [6, Theorem 9.33] in general), the CAT(0) space $X$ is either a visibility space or it contains a 2-flat $F$. Since in the former case, $G$ is Gromov-hyperbolic, we assume that $X$ contains a 2-flat $F$. In particular, $X$ contains nontrivial parallel sets.

Lemma 20. Suppose that $P=Y \times \mathbb{R}$ is a nontrivial parallel set in $X$. Then $\partial_{\infty} P$ contains a topological circle $S$ which is geodesic in the Tits metric so that $S$ bounds a disk in $\partial_{\infty} X \backslash \partial_{\infty} P$.

Proof. Let $\xi, \eta \in \partial_{\infty} P$ be the ideal points of a geodesic $y \times \mathbb{R} \subset Y \times \mathbb{R}=P$. Then the Tits boundary $\partial_{\text {Tits }} P$ is the metric join $S^{0} \star \partial_{\text {Tits }} Y$, which is the union of geodesic segments $L_{\mu}$ of length $\pi$ connecting $\eta$ and $\xi$ and passing through $\mu \in \partial_{\text {Tits }} Y \subset \partial_{\text {Tits }} X$. Clearly, if $\mu \neq \mu^{\prime}$ then $S:=L_{\mu} \cup L_{\mu^{\prime}}$ is a topological circle which is geodesic in the Tits metric.

Let $D$ be a component of $\partial_{\infty} X \backslash \partial_{\infty} P$. Then there is a point $\zeta \in \partial D$ which belongs to $L_{\mu} \backslash\{\xi, \eta\}$ for some $\mu \in \partial_{\text {Tits }} Y$. Clearly, $\partial D$ is not contained in $L_{\mu}$, therefore there exists a point $\zeta^{\prime} \in \partial D$ which belongs to $L_{\mu^{\prime}} \backslash\{\xi, \eta\}$ for some $\mu^{\prime} \in \partial_{\text {Tits }} Y \backslash\{\mu\}$. The reader will verify that the circle $S=L_{\mu} \cup L_{\mu^{\prime}}$ bounds $D$.

We will refer to these circles $S$ as in Lemma 20, as peripheral circles of $\partial_{\infty} P$. A flat in $X$ whose boundary is a peripheral circle will be called a peripheral flat.

It follows from the properties of the Tits metric (discussed in Section 2) that if $F, F^{\prime} \subset X$ are 2-flats then the intersection $\partial_{\text {Tits }} F \cap \partial_{\text {Tits }} F^{\prime} \subset \partial_{\text {Tits }} X$ is convex and either consists of two antipodal points or is a circular arc in $\partial_{\text {Tits }} F$ of the length $\leq \pi$.

Definition 21. We say that totally-geodesic circles $S, S^{\prime} \subset Z$ cross if $S$ contains points from each component of $Z \backslash S^{\prime}$ (in the visual topology). Note that crossing is a symmetric relation. We will say that the ideal boundaries of two parallel sets $P, P^{\prime}$ cross if at least one circle in $\partial_{\text {Tits }} P$ crosses a circle in $\partial_{\text {Tits }} P^{\prime}$. 
Observe that if $S$ and $S^{\prime}$ cross, the intersection $S \cap S^{\prime}$ consists of a pair of antipodal points.

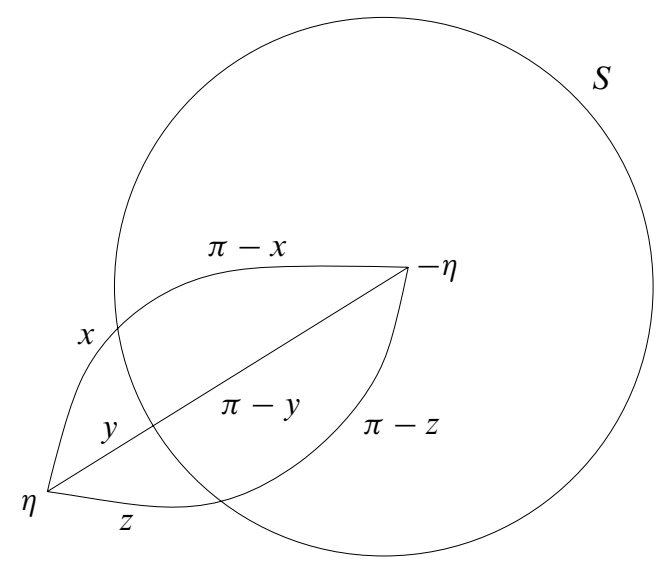

Figure 1

Lemma 22. Suppose that $P=l \times Y \subset X$ is a parallel set for which $\partial_{\infty} Y$ consists of at least 3 points (i.e., $P$ is not within finite Hausdorff distance from a flat) and $F \subset X$ is a 2-flat which is not contained in $P$. Then $\partial_{\infty} P$ and $S=\partial_{\infty} F$ do not cross.

Proof. Suppose to the contrary that $\partial_{\infty} P$ and $S=\partial_{\infty} F$ do cross. Recall that $\partial_{\infty} P$ is the metric join of $\{\eta,-\eta\}=\partial_{\infty} l$ and $\partial_{\infty} Y$. If $S$ were to pass through $\eta$ then, by convexity, $S$ passes through $-\eta$ as well and hence $F$ would be contained in the parallel set $P$. Therefore, $S$ does not pass through $\partial_{\infty} l$ and the configuration $\left\{\partial_{\infty} P, S\right\}$ has to look like the one in Figure 1, where $x, y, z$ denote the distances from $\eta$ to the points of intersection between $\partial_{\infty} P$ and $S$. It follows that $x+y=\pi, y+z=\pi$, $x+z=\pi$ and thus

$$
x=y=z=\pi / 2 .
$$

This implies that the circle $S$ is contained in $\partial_{\infty} Y$, thus $Y$ cannot be Gromovhyperbolic. This contradicts Lemma 14.

We observe that, since $G \curvearrowright X$ is properly discontinuous, the stabilizer of each flat $F \subset X$ in the group $G$ is virtually abelian. We assume that this stabilizer is virtually cyclic (possibly finite) - otherwise $G$ contains $\mathbb{Z}^{2}$.

Suppose that we have three flats $F, F^{\prime}, F^{\prime \prime} \subset X$ with pairwise distinct ideal boundaries. We will say that $F^{\prime}$ separates $F$ from $F^{\prime \prime}$ if the following holds:

$$
\partial_{\infty} F \subset \bar{D}, \partial_{\infty} F^{\prime \prime} \subset \bar{D}^{\prime \prime},
$$


where $D \sqcup D^{\prime \prime}=Z \backslash \partial_{\infty} F^{\prime}$. We set the ternary relation $\beta$ by: $\beta\left(F F^{\prime} F^{\prime \prime}\right)$ if $F^{\prime}$ separates $F$ from $F^{\prime \prime}$.

We leave it to the reader to verify that with this ternary relation the set $\mathcal{P}$ of all peripheral flats in $X$ satisfies the axioms of a pretree.

Lemma 23. If $U_{0}$ is a horoball in $X$ then $W:=\partial_{\infty} U_{0}$ does not separate $\partial_{\infty} X$.

Proof. Let $\xi \in \partial_{\infty} X$ and consider the horoballs $U_{t}=\left\{b_{\xi}(x) \leq t\right\}, t \in \mathbb{R}$, where $b_{\xi}$ is the appropriately normalized Busemann function at $\xi$. Clearly $\partial_{\infty} U_{t}=W$ for each $t$. Property (2) of the Z-set compactification applied to the pairs $\left(U_{t}, W\right)$ means that we have natural isomorphisms

$$
H_{c}^{i}\left(U_{t}\right) \rightarrow \tilde{H}^{i-1}(W) .
$$

Suppose that $[\zeta] \in H_{c}^{i}\left(U_{t}\right)$. Then there exists $s<t$ such that $U_{s}$ is disjoint from the support set of the cocycle $\zeta$. Therefore [ $\zeta]$ maps trivially to $H_{c}^{i}\left(U_{s}\right)$ and hence, by naturality of (24), it maps trivially to $\widetilde{H}^{i-1}(W)$. We conclude that $\widetilde{H}^{*}(W)=0$. Therefore, by the Alexander duality on $\partial_{\infty} X$, the subset $W=\partial_{\infty} U_{0}$ cannot separate $\partial_{\infty} X$.

Proposition 25. Let $F, F^{\prime \prime}$ be flats in $X$. Then the set $S\left(F, F^{\prime \prime}\right)$ offlats $F^{\prime}$ separating $F$ from $F^{\prime \prime}$ is compact with respect to the Gromov-Hausdorff topology.

Proof. If $\partial_{\infty} F=\partial_{\infty} F^{\prime \prime}$ then for each flat $F^{\prime}$ separating $F$ and $F^{\prime \prime}$ we have: $\partial_{\infty} F^{\prime}=$ $\partial_{\infty} F$. Therefore, $S\left(F, F^{\prime \prime}\right)$ is compact by Corollary 13 .

Whence we can assume that $\partial_{\infty} F^{\prime} \neq \partial_{\infty} F^{\prime \prime}$. Suppose that $F_{i}$ is a sequence of 2-flats in $X$ which diverge to infinity, i.e.,

$$
\lim _{i} d\left(o, F_{i}\right)=\infty
$$

where $o \in X$ is a base-point. Then, as in the proof of Lemma 10, the limit of the distance functions to $F_{i}$ (normalized at $o$ ) subconverge to a Busemann function $b_{\xi}$ in $X$. Let $U$ be the horoball $\left\{x: b_{\xi}(x) \leq 0\right\}$.

If, say, $\partial_{\infty} F \subset \partial_{\infty} U$ then the flat $F$ is contained in the sublevel set of the Busemann function $b_{\xi}$ and therefore $X$ would contain a flat half-space $\mathbb{R}_{+}^{3}$, which contradicts Lemma 12. Thus both complements

$$
\partial_{\infty} F \backslash \partial_{\infty} U, \quad \partial_{\infty} F^{\prime \prime} \backslash \partial_{\infty} U
$$

are nonempty.

Lemma 26. 1. In the Hausdorff topology on the set of closed subsets of $X \cup \partial_{\infty} X$, the sets $F_{i} \cup \partial_{\infty} F_{i}$ subconverge into $\partial_{\infty} U$.

2. $\partial_{\infty} F \cap \partial_{\infty} F^{\prime \prime} \subset \partial_{\infty} U$. 
Proof. 1. Suppose that the assertion is false. Then there exists a sequence of points $x_{i} \in \partial_{\infty} F_{i}$ such that

$$
\eta=\lim _{i} x_{i} \notin \partial_{\infty} U .
$$

Clearly, $\eta \in \partial_{\infty} X$. Consider a parametrization $\rho(t), t \in \mathbb{R}_{+}$of the geodesic ray $\overline{o \eta}$. Then, since $\eta \notin \partial_{\infty} U$, there exists $T \geq 0$ such that

$$
b_{\xi}(\rho(t)) \geq 1 \quad \text { for all } t \geq T .
$$

The Busemann function $b_{\xi}$ is the limit of the normalized distance functions

$$
d_{i}(x)=d\left(x, F_{i}\right)-d\left(o, F_{i}\right) .
$$

Then $d_{i}(o)=0, d_{i}\left(x_{i}\right) \leq 0$ for all $i$ and hence, by convexity,

$$
d_{i}\left(y_{i}\right) \leq 0 \quad \text { for all } y_{i} \in \overline{o x_{i}} .
$$

This, together with the inequality (27), contradicts the assumption that the geodesics $\overline{o x_{i}}$ converge to the geodesic ray $\overline{o \eta}$.

2. Observe that $\partial_{\infty} F \cap \partial_{\infty} F^{\prime \prime} \subset \partial_{\infty} F_{i}$ for each $i$. Thus (2) follows from (1).

We continue the proof of Proposition 25. Pick points

$$
\eta \in \partial_{\infty} F \backslash \partial_{\infty} U, \quad \eta^{\prime \prime} \in \partial_{\infty} F^{\prime \prime} \backslash \partial_{\infty} U .
$$

The previous lemma implies that

$$
\eta, \eta^{\prime \prime} \notin \partial_{\infty} F \cap \partial_{\infty} F^{\prime \prime}
$$

and that (since $\partial_{\infty} U$ does not separate $\partial_{\infty} X$ ) for large $i$ the points $\eta, \eta^{\prime \prime}$ belong to the same connected component of $\partial_{\infty} X \backslash \partial_{\infty} F_{i}$. This contradicts the assumption that $F_{i}$ is between $F, F^{\prime \prime}$ for all $i$.

Now, let us pick a peripheral 2-flat $F_{0} \in \mathcal{P}$, consider the set $\left\{g F_{0}, g \in G\right\}$ and its closure $\mathcal{F}$ in the Gromov-Hausdorff topology. The elements of $\mathcal{F}$ are peripheral 2 -flats in $X$ and the group $G$ acts naturally on $\mathcal{F}$. We note that since no flat in $\mathcal{F}$ has cocompact stabilizer, $\mathscr{F}$ contains no isolated points. After passing to a smaller $G$-invariant subset in $\mathscr{F}$ we may assume that the action $G \curvearrowright \mathscr{F}$ is minimal. The union

$$
\widetilde{\mathscr{L}}:=\cup_{F \in \mathcal{F}} F
$$

equipped with the Gromov-Hausdorff topology becomes a locally compact 2-dimensional lamination, the topological action $G \curvearrowright \widetilde{\mathscr{L}}$ is properly discontinuous and cocompact. The lamination $\widetilde{\mathscr{L}}$ has a continuous $G$-invariant leafwise flat metric. Therefore, since each leaf of $\widetilde{\mathscr{L}}$ is amenable, Plante's construction (see [17]) implies existence of a transversal $G$-invariant measure $\mu$ on $\widetilde{\mathscr{L}}$; minimality of $G \curvearrowright \mathscr{F}$ implies that this measure has full support. 
Lemma 28. Suppose that $F \in \mathcal{F}, g_{n} \in G$ is a sequence such that $\lim _{n \rightarrow \infty} g_{n} F=$ $F_{\infty} \in \mathcal{F}$. Then there exist $x_{-}, x_{+} \in \mathcal{F}$ such that for all sufficiently large $n, g_{n} F \in$ $\left[x_{-}, x_{+}\right]$and $F_{\infty} \in\left[x_{-}, x_{+}\right]$.

Proof. Since $\lim _{n \rightarrow \infty} g_{n} F=F_{\infty}$, the circles $\partial_{\text {Tits }}\left(g_{n} F\right)$ converge to the circle $\partial_{\text {Tits }} F_{\infty}$ in the Chabauty topology (we again are using here the visual topology on $Z$ ). The circles in the collection

$$
\left\{\partial_{\text {Tits }}\left(g_{n} F\right), \partial_{\text {Tits }} F_{\infty}, n \in \mathbb{N}\right\}
$$

are all peripheral and hence do not cross each other (by Lemma 22). This implies that for all large $n, m$ either $\partial_{\text {Tits }}\left(g_{n} F\right)$ separates $\partial_{\text {Tits }}\left(g_{m} F\right)$ from $\partial_{\text {Tits }} F_{\infty}$ or $\partial_{\text {Tits }} F_{\infty}$ separates $\partial_{\text {Tits }}\left(g_{n} F\right)$ from $\partial_{\text {Tits }}\left(g_{m} F\right)$.

The above lemma implies that the natural projection $p: \tilde{\mathscr{L}} \rightarrow \mathcal{F}$ is continuous, where we give $\mathcal{F}$ the order topology, whose basis consists of the open intervals $(a, b)$. It is also clear that $p$ is a proper map in the sense that for each interval $[a, b]$ the inverse image $p^{-1}([a, b])$ consists of leaves of $\widetilde{\mathscr{L}}$ which intersect a certain compact subset in $X$ : If a sequence of flats $F_{j}$ leaves every compact subset in $X$ then this sequence subconverges to a point in $\partial_{\infty} X$, but a point cannot separate one circle in $\partial_{\text {Tits }} X$ from another.

The measure $\mu$ on the pretree $\mathcal{F}$ has no atoms and (since the measure $\mu$ transversal to $\tilde{\mathscr{L}}$ has full support) for each pair of distinct points $x, x^{\prime} \in \mathcal{F}, \mu\left(\left[x, x^{\prime}\right]\right)=0$ iff the corresponding flats $F, F^{\prime}$ in $X$ are not separated by any flat in $\mathscr{F}$. We let $T$ be the quotient of $\mathcal{F}$ by the equivalence relation: Points $x, x^{\prime} \in \mathcal{F}$ are equivalent iff $\mu\left(\left[x, x^{\prime}\right]\right)=0$. The $G$-action, the pretree structure, and the measure $\mu$ project to $T$ (we retain the notation $\mu$ for the projection of the measure). As it was explained in Section 3 , the measure $\mu$ yields a metric $d$ on $T$. Local compactness of $\widetilde{L}$ implies that the restriction of $d$ to each interval in $T$ is a complete metric. It is clear that the group $G$ acts isometrically on $T$.

Remark 29. The map $\mathscr{F} \rightarrow T$ has at most countable multiplicity. Moreover, all but countably many points in $T$ have a unique preimage in $\mathscr{F}$.

Lemma 30. 1. T is an uncountable metric tree.

2. Stabilizers of nondegenerate arcs in $T$ are virtually cyclic and the action $G \curvearrowright T$ is stable.

3. $G$ does not have a global fixed point in $T$.

Proof. 1. Follows from Lemma 8.

2. By our hypothesis, for each point $F \in \mathcal{F}$ its $G$-stabilizer is virtually cyclic. Since $\mathcal{F}$ is prefect, it is uncountable; hence, by Remark 29 , uncountably many points in each nondegenerate arc $[x, y] \subset T$ have a virtually cyclic stabilizer. Thus the 
action $G \curvearrowright T$ is small. Since $G$ is a CAT(0)-group, each virtually cyclic subgroup of $G$ is contained in a maximal virtually cyclic subgroup. Therefore, if $I_{1} \supset I_{2} \supset \cdots$ is a descending chain of $\operatorname{arcs}$ in $T$, then the sequence of their stabilizers in the group $G$

$$
G_{I_{1}} \subset G_{I_{2}} \subset \cdots
$$

is eventually constant. Thus the action $G \curvearrowright T$ is stable.

3. The action $G \curvearrowright \mathcal{F}$ is minimal, hence the action $G \curvearrowright T$ is minimal as well. Since $T$ is not a point it follows that $G$ cannot fix a point in $T$.

Since $G$ acts properly discontinuously and cocompactly on the contractible space $X$, this group is finitely-presented. Therefore, by Lemma 30, we can apply [3] to conclude that the group $G$ splits as an amalgam with a virtually solvable edge subgroup $A$. Since $G$ is a CAT(0)-group, the subgroup $A$ is virtually abelian and finitely generated; let $A^{\prime} \subset A$ be a finite index free abelian subgroup. Since $G$ splits over $A$, the pair $(G, A)$ has at least two ends, and hence the same is true for the pair $\left(G, A^{\prime}\right)$. Since $G$ is a 3-dimensional Poincaré duality group over $\mathcal{R}$ this implies that $A^{\prime}$ has rank at least 2 . This proves the main theorem.

\section{References}

[1] W. Ballmann, Lectures on spaces of nonpositive curvature. DMV Sem. 25, Birkhäuser, Basel 1995. Zbl 0834.53003 MR 1377265

[2] M. Bestvina, Local homology properties of boundaries of groups. Michigan Math. J. 43 (1996), 123-139. Zbl 0872.57005 MR 1381603

[3] M. Bestvina and M. Feighn, Stable actions of groups on real trees. Invent. Math. 121 (1995), 287-321. Zbl 0837.20047 MR 1346208

[4] R. Bieri, Homological dimension of discrete groups. Queen Mary College Mathematics Notes, London 1976. Zbl 0357.20027 MR 0466344

[5] B. H. Bowditch and J. Crisp, Archimedean actions on median pretrees. Math. Proc. Cambridge Philos. Soc. 130 (2001), 383-400. Zbl 1034.20022 MR 1816800

[6] M. R. Bridson and A. Haefliger, Metric spaces of non-positive curvature. Grundlehren Math. Wiss. 319, Springer-Verlag, Berlin 1999. Zbl 0988.53001 MR 1744486

[7] K. Brown, Cohomology of groups. Grad. Texts in Math. 87, Springer-Verlag, New York 1982. Zbl 0584.20036 MR 0672956

[8] S. Buyalo, Euclidean planes in three-dimensional manifolds of nonpositive curvature. Mat. Zametki 43 (1988), 103-114; Math. Notes (1988) 43, 60-66. Zbl 0644.53035 MR 0932905

[9] H. Cartan and S. Eilenberg, Homological algebra. Princeton University Press, Princeton 1999. Zbl 0933.18001 MR 1731415

[10] A. Casson and D. Jungreis, Convergence groups and Seifert fibered 3-manifolds. Invent. Math. 118 (1994), 441-456. Zbl 0840.57005 MR 1296353 
[11] P. Eberlein, Geodesic flow on certain manifolds without conjugate points. Trans. Amer. Math. Soc. 167 (1972),151-170. Zbl 0209.53304 MR 0295387

[12] D. Gabai, Convergence groups are Fuchsian groups. Ann. of Math. (2) 136 (1992), 447-510. Zbl 0785.57004 MR 1189862

[13] A. Hinkkanen, Abelian and nondiscrete convergence groups on the circle. Trans. Amer. Math. Soc. 318 (1990), 87-121. Zbl 0699.30017 MR 1000145

[14] M. Kapovich and B. Kleiner, Geometry of quasi-planes. Preprint 2004.

[15] M. Kapovich and B. Leeb, Quasi-isometries preserve the geometric decomposition of Haken manifolds. Invent. Math. 128 (1997), 393-416. Zbl 0866.20033 MR 1440310

[16] L. Mosher, Geometry of cubulated 3-manifolds. Topology 34 (1995), 789-814. Zbl 0869.57015 MR 1362788

[17] J. Plante, Foliations with measure preserving holonomy. Ann. of Math. (2) 102 (1975), 327-361. Zbl 0314.57018 MR 0391125

[18] V. Schroeder, Codimension one tori in manifolds of nonpositive curvature. Geom. Dedicata 33 (1990), 251-265. Zbl 0698.53026 MR 1050413

[19] P. Tukia, Homeomorphic conjugates of Fuchsian groups. J. Reine Angew. Math. 391 (1988), 1-54. Zbl 0644.30027 MR 0961162

Received May 11, 2006; revised September 15, 2006

Department of Mathematics, University of California, Davis, CA 95616, U.S.A.

E-mail: kapovich@math.ucdavis.edu

Department of Mathematics, Yale University, New Haven, CT 06520-8283, U.S.A.

E-mail: bruce.kleiner@yale.edu 